\title{
Change in Augmentation Index during NOS Inhibition, an Index of Basal NO Production, Is an Independent Determinant of Large-Artery Function
}

\author{
M.P. Schneider C. Ott U. Raff M. Ritt R.E.Schmieder \\ Department of Nephrology and Hypertension, University of Erlangen-Nuremberg, Erlangen-Nuremberg, Germany
}

\section{Key Words}

Nitric oxide $\cdot$ Pulse $\cdot$ Arteries $\cdot$ Endothelium •

Endothelium-dependent relaxing factors

\begin{abstract}
Background/Aims: Arterial wave reflection, measured as augmentation index (Alx), and central pulse pressure (PP) closely predict cardiovascular events. We hypothesized that basal nitric oxide (NO) production would be a determinant of Alx and central PP. Methods: Alx and central PP were assessed at baseline by pulse wave analysis in 86 male subjects across a wide range of age, blood pressure and lipid values. The basal NO production in the cardiovascular system was then determined as change in Alx during NO synthase blockade with $\mathrm{N}^{\mathrm{G}}$-monomethyl-L-arginine (L-NMMA, $3.25 \mathrm{mg} / \mathrm{kg}$ ). Results: Alx increased from $17.5 \pm 14.6$ to $23.1 \pm 14.2$ during L-NMMA infusion $(p<0.001)$. The increase in Alx during NO synthase blockade, an index of basal NO production, was inversely related to baseline central Alx and PP, and positively to PP amplification. Multiple linear regression analyses disclosed that in addition to age and mean blood pressure, change of Alx to L-NMMA is a strong and independent determinant of baseline central Alx, central PP and PP amplification. Conclusion: Greater change of Alx to L-NMMA, an index of basal NO production, is associated with better large-ar-
\end{abstract}

tery function. Therefore, therapeutic interventions which increase the basal NO production might be particularly effective in reducing cardiovascular risk.

Copyright $\odot 2010$ S. Karger AG, Basel

\section{Introduction}

Previous studies have shown that the diastolic blood pressure (BP) slightly decreases but, more strikingly, the systolic BP increases from central towards peripheral vessels in young, healthy subjects [1]. This amplification of systolic BP is mainly the result of superimposed pulse wave reflections from distal reflection sites on the incident pulse wave traveling from the aorta towards the peripheral vasculature. Furthermore, this phenomenon explains why the peripheral pulse pressure (PP) is higher than the central PP in young, healthy subjects (natural 'pulse pressure amplification'). Wave reflections from peripheral sites traveling 'backwards' to the aorta are of relatively low amplitude and speed in young and healthy subjects and therefore reach the aorta during diastole, which may have favorable effects on diastolic perfusion of coronary arteries.

In elderly subjects and in patients with atherosclerosis, an increased pulse wave velocity (PWV) due to arterial

\section{KARGER \\ Fax +41613061234 E-Mail karger@karger.ch} www.karger.com
Roland E. Schmieder, MD

Department of Nephrology and Hypertension, University of Erlangen-Nuremberg Krankenhausstrasse 12, DE-91054 Erlangen (Germany)

Tel. +49 913185 36245, Fax +49 91318539209

E-Mail roland.schmieder@ rzmail.uni-erlangen.de 
stiffening and an increased amplitude of wave reflections due to peripheral vasoconstriction lead to an earlier return and a higher amplitude of arterial wave reflections in the aorta. The subsequent augmentation of central systolic BP and PP is associated with loss of PP amplification, meaning that the central and peripheral PP become more similar. The central PP therefore depends on the structure and function of large and small arteries and cannot be predicted from peripheral PP alone. With computerized pulse wave analysis, a noninvasive tool to study arterial wave reflection (central augmentation index, AIx) and central PP has become available. Using this innovative approach, the independent predictive value of arterial wave reflection and central PP for cardiovascular outcome has been demonstrated $[2,3]$. Furthermore, recent studies have shown substantial differences in the ability of antihypertensive drugs to affect these parameters and cardiovascular risk $[4,5]$.

The importance of nitric oxide (NO) for intact vascular function, in particular provided by its vasodilatory and antiproliferative properties, has been thoroughly documented. We therefore hypothesized that NO modulates arterial wave reflection and central hemodynamics. In humans, a recent study has shown that endothelial function, determined either by the decrease in AIx during inhalation of the endothelium-dependent vasodilator albuterol or by brachial artery flow-mediated vasodilation, is associated with PWV, AIx and central PP [6]. However, both of these investigations are tests of stimulated endothelial function and do not reflect the basal contribution of NO to vascular tone. Basal NO production and stimulated endothelial function are not interchangeable, and a number of previous studies have shown that these can be affected disparately by antihypertensive drugs $[7,8]$.

Analogous to analyzing stimulated endothelial function via the decrease in AIx during inhalation of albuterol, the increase in AIx during competitive NO synthase (NOS) blockade can be regarded as an index of basal NO production [9]. In the current study, we used this approach to examine the relationship between basal $\mathrm{NO}$ production and central hemodynamics in humans.

\section{Methods}

\section{Subjects}

A total number of 86 subjects across a wide range of age, BP and lipid values were recruited by advertisement in the local newspapers. Only male subjects were included in this study because of profound gender differences in timing of arterial wave reflections. Further exclusion criteria were type 1 or type 2 diabetes, renal disease, clinically relevant liver disease, clinically manifest cardiovascular disease as reflected by a previous myocardial infarction, a coronary intervention procedure, stroke or peripheral vascular disease and current treatment with any type of antihypertensive or lipid-lowering medication. The study was approved by the local University Research Ethics Committee and conducted according to the Declaration of Helsinki and Good Clinical Practice Guidelines. Written informed consent for participation in the study was obtained from each individual.

Measurement of Arterial Wave Reflections and Central PP

Brachial BP was recorded after 30 min rest in the supine position in the dominant arm using a validated ocillometric device (Dinamap Compact T; Johnson \& Johnson Medical Ltd., Newport, UK). The BP was measured 5 times over $10 \mathrm{~min}$ and the mean of the last 3 measurements was taken for calibration. To derive the central arterial waveform, a validated system (Sphygmocor ${ }^{\mathrm{TM}}$; AtCor Medical, Sydney, Australia) was used that employs high-fidelity applanation tonometry (Millar) for noninvasive registration of peripheral arterial pressure waves and appropriate computer software for pressure wave analysis (Sphygmocor ${ }^{\mathrm{TM}}$ ) [10].

Pressure wave recording was performed at the radial artery of the same arm with the wrist gently hyperextended. The pressure wave was averaged from single pressure waves recorded consecutively for $10 \mathrm{~s}$. Averaged pressure waves were accepted only if the variation in peak and bottom pressures of single pressure waves was $<5 \%$. The central pressure wave was then automatically synthesized from the radial pressures by a built-in generalized transfer function [10]. From the derived central waveforms, data are given on central systolic and diastolic BP, central PP, augmentation pressure (AP) as the pressure height difference between second and first systolic peaks and the central AIx defined as the pressure difference between these peaks, expressed as a percentage of central PP. AP and AIx are also given normalized to a heart rate of 75 beats/min $\left(\mathrm{AP}_{75}\right.$ and $\mathrm{AIx}$ 75). $\mathrm{PP}$ amplification is defined by the ratio of peripheral $\mathrm{PP}$ to central $\mathrm{PP}$.

\section{Assessment of Basal Vascular NO Production}

The change in AIx during administration of the competitive NOS inhibitor $\mathrm{N}^{\mathrm{G}}$-monomethyl-L-arginine (L-NMMA) was determined as an index of basal NO production [9]. After assessing baseline hemodynamics as described in the section above, L-NMMA was administered intravenously as a bolus infusion ( $3 \mathrm{mg} / \mathrm{kg}$ body weight) over $5 \mathrm{~min}$ followed by constant infusion $(0.05 \mathrm{mg} / \mathrm{kg} / \mathrm{min})$ over a further $5-\mathrm{min}$ period. The total dose of L-NMMA was $3.25 \mathrm{mg} / \mathrm{kg}$ body weight. During the last $3 \mathrm{~min}$ of infusion, the measurements of arterial wave reflections and central PP were repeated and the basal NO production was defined as the change in AIx between the measurements during L-NMMA administration and baseline. For safety reasons, we subsequently infused $100 \mathrm{mg} / \mathrm{kg}$ body weight of L-arginine (L-arginine hydrochloride 6\%, University Hospital Pharmacy, Erlangen, Germany) over $30 \mathrm{~min}$ to counteract L-NMMA-induced vasoconstriction (data not shown).

eNOS Genotyping

Genomic DNA was extracted from 2-5 $\mathrm{ml}$ of whole blood by standard methods, using a commercially available kit (Flexi Gene DNA Kit 250; QIAGEN GmbH, Hilden, Germany). The eNOS 
Glu298Asp (298G/T) polymorphism was determined using the TaqMan system (TaqMan SNP Genotyping Assays; Applied Biosystems, Foster City, Calif., USA). The investigator who assessed the genotype was blind to the clinical data of the patients from whom the samples originated.

\section{Statistics}

Parametric distribution of data was confirmed by the Kolmogorov-Smirnov test before further analysis. Parametric clinical baseline data were compared by unpaired Student's t tests and nonparametric data by Mann-Whitney U tests. Analysis of vari-

Table 1. Clinical data of the study population $(n=86)$ at baseline

\begin{tabular}{lc}
\hline Parameter & Mean \pm SD \\
\hline Age, years & $43.0 \pm 12.7(20-72)$ \\
Weight, kg & $83 \pm 13(60-124)$ \\
Height, cm & $179 \pm 9(155-198)$ \\
BMI, kg/m & $25.8 \pm 3.6(19.6-40.3)$ \\
Total cholesterol, mg/dl & $246 \pm 51(111-350)$ \\
HDL cholesterol, mg/dl & $54 \pm 13(36-77)$ \\
LDL cholesterol, mg/dl & $157 \pm 38(60-255)$ \\
Triglycerides, mg/dl & $165 \pm 97(48-536)$ \\
Creatinine, mg/dl & $0.9 \pm 0.1(0.8-1.2)$ \\
Glucose, mg/dl & $97 \pm 8(57-123)$ \\
Hs-CRP, mg/l & $1.7 \pm 2.4(0.15-10.7)$ \\
UACR, mg/g creatinine & $8.6 \pm 5.7(2.3-21.5)$ \\
\end{tabular}

Figures in parentheses are ranges. BMI = Body mass index; $\mathrm{HDL}$ = high-density lipoprotein; LDL = low-density lipoprotein; Hs-CRP = high-sensitivity C-reactive protein; UACR = urinary albumin excretion rate (spot urine). ance (ANOVA) was used to compare data between groups stratified according to tertiles of basal NO production. Pearson's correlation coefficients for parametric and Spearman correlation coefficients for nonparametric data were given when indicated. Multiple linear stepwise regression analyses were conducted to identify the variables independently predicting parameters of arterial wave reflection, central PP and PP amplification. Twotailed $\mathrm{p}$ values $<0.05$ were considered statistically significant. Data in the text and in the tables are given as means \pm standard deviation (SD).

\section{Results}

The clinical characteristics of the study population are given in table 1 . Male subjects with a wide range of age, weight, body mass index, cholesterol and peripheral BP values were included. During infusion of L-NMMA, the peripheral systolic and diastolic BP increased, while the peripheral PP did not change (table 2). In contrast, the central PP increased during L-NMMA infusion, which was associated with a decrease in central to peripheral PP amplification and an increase in central AIx.

The subjects were classified into tertiles according to the increase in AIx during L-NMMA infusion, as an index of basal vascular NO production. Subsequently, the relationships between tertiles of basal NO production and baseline (pre-L-NMMA) peripheral and central pulse wave characteristics were examined. No differences were detected between tertiles of this index of basal NO production in baseline peripheral pulse wave characteristics,

Table 2. Effect of NOS blockade $(3.25 \mathrm{mg} / \mathrm{kg})$ on pulse wave characteristics

\begin{tabular}{lcrc}
\hline Parameter & Before L-NMMA & During L-NMMA & p value \\
\hline Peripheral systolic BP, mm Hg & $126 \pm 15$ & $131 \pm 16$ & $<0.001$ \\
Peripheral diastolic BP, mm Hg & $78 \pm 10$ & $83 \pm 12$ & $<0.001$ \\
Peripheral MAP, mm Hg & $94 \pm 12$ & $99 \pm 12$ & $<0.001$ \\
Peripheral PP, mm Hg & $48 \pm 12$ & $48 \pm 12$ & n.s. \\
Central systolic BP, mm Hg & $114 \pm 16$ & $121 \pm 17$ & $<0.001$ \\
Central diastolic BP, mm Hg & $79 \pm 11$ & $84 \pm 12$ & $<0.001$ \\
Central PP, mm Hg & $35 \pm 11$ & $38 \pm 13$ & 0.005 \\
HR, beats/min & $64 \pm 9$ & $57 \pm 9$ & $<0.001$ \\
AP, mm Hg & $7.1 \pm 7.3$ & $9.6 \pm 8.3$ & $<0.001$ \\
AP at HR & & \\
AIx, $\%$ & $4.6 \pm 6.2$ & $5.5 \pm 6.2$ & 0.024 \\
AIx at $\mathrm{HR}_{75}, \%$ & $18 \pm 15$ & $23 \pm 14$ & $<0.001$ \\
PP amplification & $12 \pm 14$ & $15 \pm 13$ & 0.010 \\
\end{tabular}

$\mathrm{MAP}=$ Mean arterial pressure; $\mathrm{PP}=$ pulse pressure; $\mathrm{HR}=$ heart rate. 
Table 3. Clinical data and pulse wave characteristics of patients classified into tertiles according to change in AIx as an index of basal NO production

\begin{tabular}{|c|c|c|c|c|}
\hline Parameter & $\begin{array}{l}\text { Low } \Delta \text { AIx } \\
(\mathrm{n}=29)\end{array}$ & $\begin{array}{l}\text { Medium } \Delta \text { AIx } \\
(\mathrm{n}=29)\end{array}$ & $\begin{array}{l}\text { High } \Delta \text { AIx } \\
(n=28)\end{array}$ & $\mathrm{p}$ value \\
\hline Age, years & $43 \pm 13$ & $44 \pm 13$ & $42 \pm 12$ & n.s. \\
\hline Weight, kg & $83 \pm 15$ & $84 \pm 16$ & $81 \pm 12$ & n.s. \\
\hline Height, cm & $178 \pm 9$ & $178 \pm 9$ & $179 \pm 9$ & n.s. \\
\hline BMI, $\mathrm{kg} / \mathrm{m}^{2}$ & $26.2 \pm 4.1$ & $25.9 \pm 3.4$ & $25.1 \pm 3.5$ & n.s. \\
\hline Total cholesterol, mg/dl & $249 \pm 47$ & $241 \pm 44$ & $243 \pm 54$ & n.s. \\
\hline HDL cholesterol, mg/dl & $55 \pm 14$ & $50 \pm 8$ & $54 \pm 14$ & n.s. \\
\hline LDL cholesterol, mg/dl & $160 \pm 35$ & $155 \pm 32$ & $153 \pm 40$ & n.s. \\
\hline Triglycerides, mg/dl & $154 \pm 99$ & $175 \pm 86$ & $158 \pm 77$ & n.s. \\
\hline Glucose, $\mathrm{mg} / \mathrm{dl}$ & $93 \pm 13$ & $100 \pm 11$ & $98 \pm 14$ & n.s. \\
\hline Creatinine, mg/dl & $0.9 \pm 0.1$ & $0.9 \pm 0.1$ & $0.9 \pm 0.1$ & n.s. \\
\hline Hs-CRP, mg/l & $1.8 \pm 2.4$ & $1.6 \pm 1.9$ & $0.9 \pm 0.9$ & n.s. \\
\hline UACR, mg/g creatinine & $6.4 \pm 2.7$ & $10.4 \pm 6.4$ & $8.1 \pm 6.2$ & n.s. \\
\hline Peripheral systolic BP, mm Hg & $126 \pm 15$ & $128 \pm 17$ & $124 \pm 15$ & n.s. \\
\hline Peripheral diastolic $\mathrm{BP}, \mathrm{mm} \mathrm{Hg}$ & $76 \pm 10$ & $78 \pm 11$ & $81 \pm 10$ & n.s. \\
\hline Peripheral MAP, mm Hg & $93 \pm 10$ & $95 \pm 14$ & $94 \pm 11$ & n.s. \\
\hline Peripheral PP, mm Hg & $50 \pm 11$ & $50 \pm 10$ & $44 \pm 13$ & 0.053 \\
\hline Central systolic BP, mm Hg & $115 \pm 13$ & $116 \pm 20$ & $110 \pm 16$ & n.s. \\
\hline Central diastolic BP, mm Hg & $77 \pm 10$ & $80 \pm 11$ & $81 \pm 10$ & n.s. \\
\hline Central PP, mm Hg & $38 \pm 8$ & $37 \pm 12$ & $30 \pm 12$ & 0.006 \\
\hline $\mathrm{HR}$, beats/min & $64 \pm 11$ & $64 \pm 9$ & $64 \pm 8$ & n.s. \\
\hline $\mathrm{AP}, \mathrm{mm} \mathrm{Hg}$ & $9.0 \pm 6.5$ & $8.0 \pm 8.2$ & $3.9 \pm 6.2$ & 0.011 \\
\hline $\mathrm{AP}$ at $\mathrm{HR}_{75}, \mathrm{~mm} \mathrm{Hg}$ & $6.2 \pm 5.0$ & $5.7 \pm 7.8$ & $1.8 \pm 4.4$ & 0.008 \\
\hline $\mathrm{AIx}, \%$ & $23 \pm 14$ & $18 \pm 14$ & $11 \pm 13$ & 0.004 \\
\hline AIx at $\mathrm{HR}_{75}, \%$ & $17 \pm 13$ & $13 \pm 14$ & $5 \pm 12$ & 0.002 \\
\hline PP amplification & $1.3 \pm 0.2$ & $1.4 \pm 0.2$ & $1.5 \pm 0.2$ & 0.005 \\
\hline
\end{tabular}

$\mathrm{BMI}=$ Body mass index; HDL = high-density lipoprotein; LDL = low-density lipoprotein; Hs-CRP = high-sensitivity C-reactive protein; $\mathrm{UACR}=$ urinary albumin excretion rate (spot urine); $\mathrm{MAP}=$ mean arterial pressure; $\mathrm{HR}=$ heart rate.

except for a trend towards a lower peripheral PP in subjects with high basal NO production (table 3). In contrast, central PP, central AP, central AP $\mathrm{P}_{75}$, central AI and central $\mathrm{AI}_{75}$, as well as $\mathrm{PP}$ amplification were significantly different between tertiles, with lower values in the groups with greater basal NO production (fig. 1 for the effects on central AIx, fig. 2 for central PP and fig. 3 for PP amplification).

As a factor potentially modulating basal NO production, the effects of the eNOS $298 \mathrm{G} / \mathrm{T}$ gene polymorphism on basal NO production and on central pulse wave characteristics were examined. However, the eNOS 298 G/T gene polymorphism did neither affect the response of AIx during L-NMMA infusion nor any pulse wave characteristics, including central PP (as an example, $\Delta$ AIx during L-NMMA infusion: $+6.1 \pm 8.1$ in GG vs. $+6.6 \pm 10.2$ in GT vs. $-0.6 \pm 10.6$ in TT allele carriers, n.s.).
Correlational analyses in the whole study group revealed that age, peripheral mean $\mathrm{BP}$ and change in AIx during L-NMMA were correlated with the parameters central PP, AP, AP $\mathrm{A}_{75}, \mathrm{AIx}, \mathrm{AIx}_{75}$ and with PP amplification (table 4). The correlations between LDL cholesterol and central PP, and between high-sensitivity C-reactive protein and PP amplification failed to reach statistical significance but were significant for the remaining parameters of central pulse wave characteristics. The inverse relationship between change in AIx during LNMMA infusion as an index of basal NO production and baseline (pre-L-NMMA) central PP is shown in figure 4 . To determine which of the univariate variables independently predict baseline central pulse wave characteristics, linear regression analyses were performed. These analyses disclosed that when all of these factors are taken into account, in addition to age and peripheral mean BP, bas- 


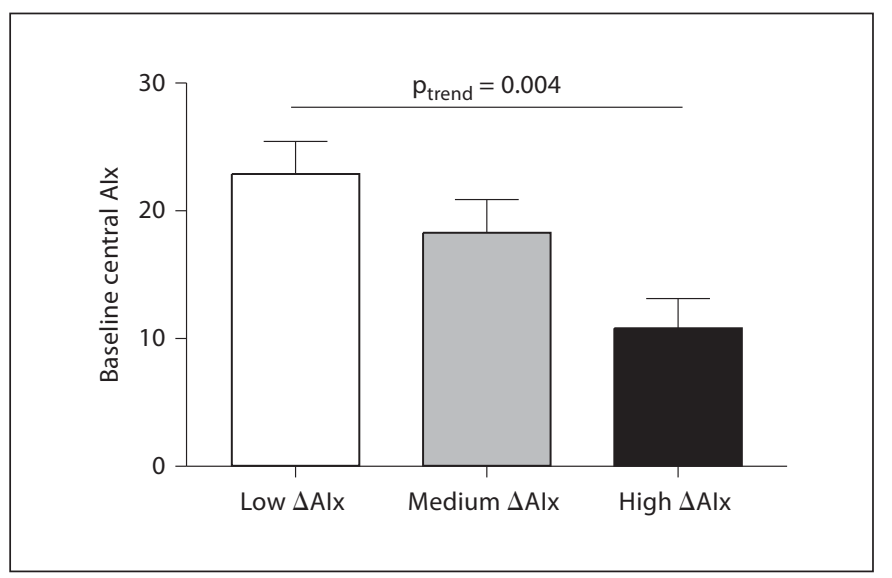

Fig. 1. Central AIx (pre-L-NMMA) in subjects classified into tertiles of low, medium and high change in AIx during L-NMMA infusion, as an index of basal NO production.

al NO production remained a strong and independent determinant of central AIx, central PP and PP amplification (table 5).

\section{Discussion}

Several previous studies in animal models demonstrated that NO modulates important determinants of arterial wave reflection. Removal of the endothelium was shown to acutely increase vascular wall viscosity and local stiffness, associated with vasoconstriction of underlying smooth-muscle cells [11, 12]. More directly assessing the role of NO, intravenous infusion of the NOS inhibitor L-NAME into rats increased PWV [13]. In addition to these acute effects, there is evidence to suggest that chronic NOS blockade increases PWV also via structural remodeling of the vessel wall $[13,14]$. A limitation of the systemic infusion of vasoconstrictors is the resulting increase in large-artery distending pressure, which directly increases PWV. Thus, a separate group conducted experiments with local administration of L-NMMA into the hindlimb circulation of sheep [15]. These results could demonstrate that L-NMMA increases PWV in the absence of a significant increase in distending BP.

In humans, several studies provided evidence for a role of NO in modulating arterial stiffness, which is a key determinant of arterial wave reflection. In a study in a large number of hypertensive subjects, peripheral PP, a marker of arterial stiffness, was the strongest independent predictor of acetylcholine-induced vasodilation in the fore-

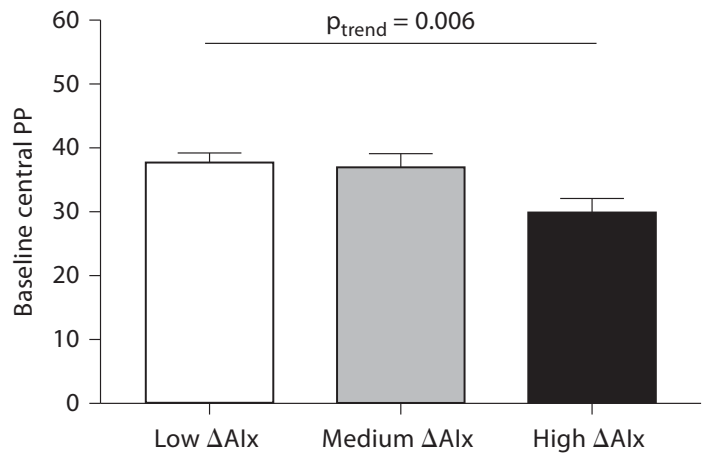

Fig. 2. Central PP (pre-L-NMMA) in subjects classified into tertiles of low, medium and high change in AIx during L-NMMA infusion, as an index of basal NO production.

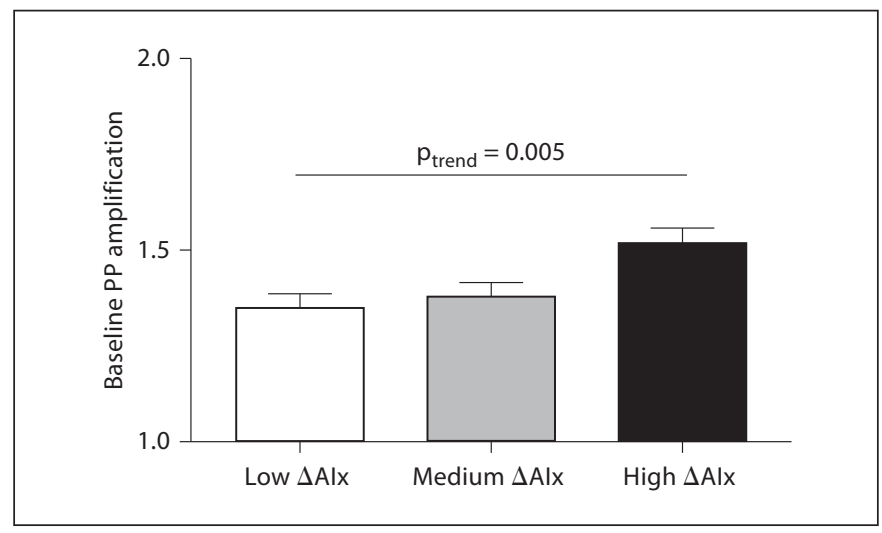

Fig. 3. PP amplification (pre-L-NMMA) in subjects classified into tertiles of low, medium and high change in AIx during L-NMMA infusion, as an index of basal NO production.

arm circulation [16]. In other studies, an inverse relationship between flow-mediated vasodilation of the brachial artery and large-artery stiffness as determined by characteristic aortic impedance and PWV was demonstrated [17-20]. Examining markers of local arterial stiffness, a separate study demonstrated that forearm postischemic vasodilation is correlated with distensibility of the carotid artery in patients with end-stage renal disease [21], and corresponding observations were made in healthy subjects [22] and in patients with heart failure [23].

So far, only 1 study examined the relationships between endothelial function, arterial wave reflections and 
Fig. 4. Relationship between change in AIx during L-NMMA infusion, as an index of basal NO production, and baseline (preL-NMMA) central PP.

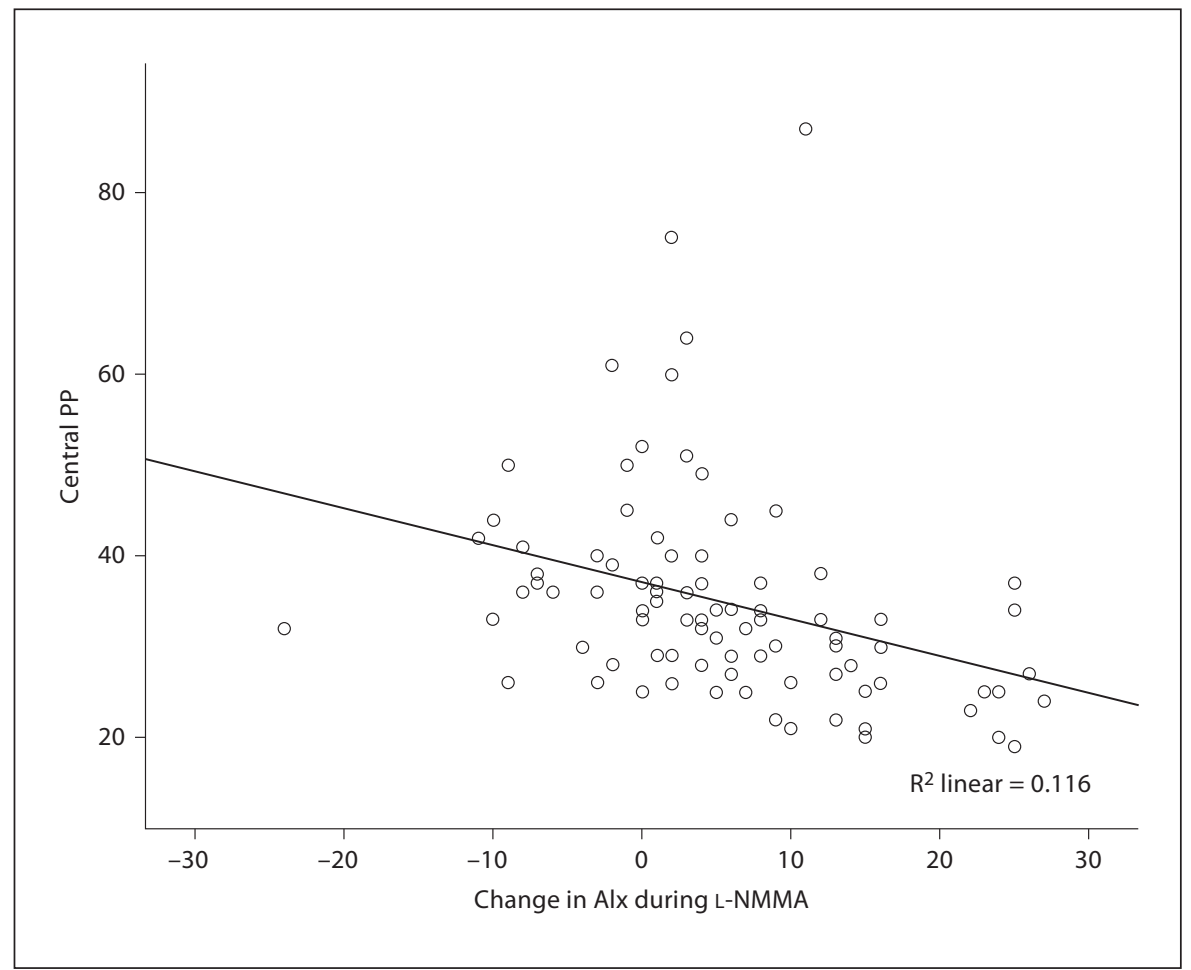

Table 4. Factors correlating with central pulse wave characteristics at baseline

\begin{tabular}{|c|c|c|c|c|c|c|c|c|c|c|}
\hline Parameter & Age & $\mathrm{p}$ & $\begin{array}{l}\text { Mean } \\
\text { BP }\end{array}$ & $\mathrm{p}$ & $\begin{array}{l}\text { LDL } \\
\text { cholesterol }\end{array}$ & $\mathrm{p}$ & Hs-CRP & $\mathrm{p}$ & $\begin{array}{l}\Delta \text { AIx to } \\
\text { L-NMMA }\end{array}$ & $\mathrm{p}$ \\
\hline Central PP & +0.480 & $<0.001$ & +0.441 & $<0.001$ & +0.164 & n.s. & +0.251 & 0.032 & -0.341 & 0.001 \\
\hline $\mathrm{AP}$ & +0.716 & $<0.001$ & +0.443 & $<0.001$ & +0.316 & 0.001 & +0.379 & 0.001 & -0.346 & 0.001 \\
\hline $\mathrm{AP}_{75}$ & +0.702 & $<0.001$ & +0.480 & $<0.001$ & +0.301 & 0.002 & +0.341 & 0.003 & -0.343 & 0.001 \\
\hline AIx & +0.714 & $<0.001$ & +0.398 & $<0.001$ & +0.346 & $<0.001$ & +0.317 & 0.007 & -0.375 & $<0.001$ \\
\hline $\mathrm{AIx}_{75}$ & +0.715 & $<0.001$ & +0.430 & $<0.001$ & +0.335 & 0.007 & +0.322 & 0.006 & -0.393 & 0.001 \\
\hline PPA & -0.707 & $<0.001$ & -0.399 & $<0.001$ & -0.351 & $<0.001$ & -0.107 & n.s. & +0.351 & 0.001 \\
\hline
\end{tabular}

$\mathrm{LDL}=\mathrm{Low}$-density lipoprotein; Hs-CRP = high-sensitivity C-reactive protein; $\Delta \mathrm{AIx}$ to L-NMMA = change in AIx in response to L-NMMA infusion; $\mathrm{AP}_{75}=$ augmentation pressure at a heart rate of 75 beats $/ \mathrm{min}$; $\mathrm{AIx}_{75}=$ augmentation index at a heart rate of 75 beats/min; PPA = pulse pressure amplification.

central pulse wave characteristics in humans. In that study, McEniery et al. [6] demonstrated that endothelial function, determined either by the decrease in AIx during inhalation of the endothelium-dependent vasodilator albuterol or by brachial artery flow-mediated vasodilation, is associated with PWV, baseline central AIx and central PP. However, both of these investigations are tests of stimulated endothelial function and do not reflect basal contribution of NO to vascular tone. Previous studies have shown that basal and stimulated endothelial function can be affected disparately by antihypertensive drugs $[7,8]$ and are thus not interchangeable. Analogous to analyzing stimulated endothelial function via the decrease in AIx during inhalation of an agent which stimulates endothelium-dependent vasodilation, the increase in AIx during competitive NOS blockade can be regarded as an index of basal NO production [9]. In the current study, we used this approach to examine the relationship between 
Table 5. Linear regression analyses

\begin{tabular}{llr}
\hline $\begin{array}{l}\text { Parameter/ } \\
\text { determinants }\end{array}$ & $\beta$-Coefficient & $\mathrm{p}$ value \\
\hline Central AIx & & \\
$\quad$ Age & +0.658 & $<0.001$ \\
$\quad$ Peripheral mean BP & +0.096 & 0.285 \\
LDL cholesterol & +0.018 & 0.841 \\
Hs-CRP & +0.134 & 0.133 \\
eNOS genotype & -0.019 & 0.833 \\
$\Delta$ AIx to L-NMMA & -0.298 & 0.001 \\
Central PP & & \\
Age & +0.215 & 0.040 \\
Peripheral mean BP & +0.383 & $<0.001$ \\
LDL cholesterol & -0.015 & 0.703 \\
Hs-CRP & +0.694 & 0.251 \\
eNOS genotype & +0.183 & 0.607 \\
$\Delta$ AIx to L-NMMA & -0.487 & $<0.001$ \\
PP amplification & & \\
Age & -0.644 & $<0.001$ \\
Peripheral mean BP & -0.102 & 0.287 \\
LDL cholesterol & -0.018 & 0.859 \\
Hs-CRP & -0.125 & 0.189 \\
eNOS genotype & -0.061 & 0.527 \\
$\Delta$ AIx to L-NMMA & +0.208 & 0.027 \\
\hline
\end{tabular}

this index of basal NO production and parameters of large-artery function.

We recruited a relatively large number of subjects with a wide range of age and cardiovascular risk factors. Competitive NOS blockade led to a significant increase in central AIx, similar to previously published data [9]. When classifying subjects into tertiles according to the increase in AIx, as an index of basal NO production, subjects with a higher NO production displayed reduced arterial wave reflections, lower central PP and greater PP amplification at baseline (pre-L-NMMA infusion). Correlational analyses revealed modest relationships between peripheral BP, LDL cholesterol, high-sensitivity C-reactive protein levels and central pulse wave characteristics. Stronger relationships were detected between age and change in AIx to L-NMMA with parameters of large-artery function. Taking all univariate variables into account, linear regression analysis disclosed that in addition to age and peripheral BP, change in AIx as an index of basal NO production is a strong and independent determinant of baseline central AIx, central PP and PP amplification.

NOS function is modulated by a variety of mechanisms, including location within the cell (membranebound vs. cytosolic), association with caveolin and phosphorylation status, but these mechanisms are difficult to study in vivo. A common gene polymorphism at amino acid position 298 resulting in a substitution of aspartate for glutamate exists. In vitro, this mutation renders eNOS more susceptible to intracellular cleavage [24], although subsequent studies have questioned whether this may simply be a methodological artifact [25]. Some studies have suggested that this mutation has functional effects in vivo, leading to reduced endothelium-dependent, NO-mediated vasodilation [26], while others, including our group, have not found any impact on the human forearm circulation $[27,28]$. In the current study, we could not detect any effect of this gene polymorphism on basal NO production or on peripheral or central pulse wave characteristics.

Our data demonstrate that in addition to stimulated endothelial function, change in AIx as an index of basal vascular NO production is a strong and independent determinant of arterial wave reflection, central PP and PP amplification. Increasing basal NO production may therefore be an attractive therapeutic goal in order to reduce arterial wave reflection, central PP and cardiovascular risk. The CAFE (conduit artery functionl evaluation) trial has demonstrated that antihypertensive therapy with a regimen based on amlodipine/perindopril is superior in lowering central AIx and PP compared to a regimen based on atenolol and a thiazide diuretic [5]. In the REASON (regression of arterial stiffness with perindopril/indapamide fixed low-dose combination) project, perindopril/ indapamide reduced arterial wave reflections and lowered the central systolic BP to a greater degree than therapy with atenolol [4]. Both of these trials have shown that greater reductions in these central vascular parameters correlate with better cardiovascular outcome. We have previously shown in 2 separate trials that arterial wave reflection is reduced to a greater extent during treatment with an angiotensin receptor blocker compared to treatment with either atenolol [29] or hydrochlorothiazide [30]. Calcium channel blockers, angiotensin-converting enzyme inhibitors and angiotensin receptor blockers have repeatedly been shown to have favorable effects on endothelium-dependent vasodilation and on basal NO production $[7,8,31-34]$. It is tempting to speculate that their ability to increase stimulated and/or basal vascular NO production contributes to their beneficial effects on arterial wave reflection and central hemodynamics. With few exceptions [35], no such positive effects on endothelial function have been demonstrated for atenolol $[31,33,36]$. It is important to note, however, that this is unlikely to be a class effect of all $\beta$-blockers considering that nebivolol has been shown to improve endothelium-dependent NO production and to reduce arterial wave reflection $[36,37]$. 
Several limitations of our study have to be acknowledged. Firstly, the dose of L-NNMA administered in the current study as a bolus has been shown to lead to significant reductions in organ perfusion including the kidney and the retina $[38,39]$. To maximize patient safety, we subsequently administered L-arginine to reverse LNMMA-induced vasoconstriction. To our knowledge, higher doses of L-NMMA for systemic administration have not been tested, and we do not know whether the dose used by us and others achieves maximum blockade of NOS. For safety reasons, we refrained from using higher doses. Secondly, pharmacological inhibition of the baroreflex by trimethaphan has recently been shown to further increase the responses of total peripheral resistance and BP to L-NMMA [40]. Again, due to safety reasons we refrained from using this approach. As another potential limitation, we acknowledge the mathematical relationship between basal NO production as defined by increase in AIx during L-NMMA and baseline (pre-LNMMA) pulse wave characteristics. As noted earlier, a previous study successfully used a similar approach to study the relationship between decrease in AIx during albuterol inhalation and baseline (prealbuterol) pulse wave characteristics [6]. Moreover, in a subgroup of patients, this study demonstrated a significant correlation between change in AIx as a systemic index of stimulated NO production and local endothelium-dependent vasodilation as determined by the increase in forearm blood flow in response to intra-arterial infusion of acetylcholine. Further, the authors found no correlation between the decrease in AIx in response to sublingual nitroglycerine and baseline large-artery function, demonstrating a specific role for the endothelium (albuterol), rather than implying abnormalities of smooth-muscle function (nitroglycerine). Further, our goal was to relate the change in AIx to L-NMMA infusion, as an index of basal $\mathrm{NO}$ production, with baseline (pre-L-NMMA) parameters of large-artery function. We appreciate that the lack of use of another vasoconstrictor agent as a control experiment, e.g. by aiming to achieve an increase in MAP similar to the one observed with L-NMMA, could also be seen as a limitation. However, because of the substantially different acute effects of other vasoconstrictors such as norepinephrine and angiotensin II on various components of cardiovascular regulation $[41,42]$, we are not sure which agent would have been most appropriate. Finally, it has to be acknowledged that production of NO is only one aspect of 'endothelial function' and that other mediators contribute to the regulation of basal vascular tone and to structural remodeling, including prostaglandins and endothelium-derived hyperpolarizing factor(s), none of which were addressed in the current study.

In conclusion, our data demonstrate that the change in AIx during L-NMMA infusion, as an index of basal NO production, is a strong and independent predictor of arterial wave reflection, central PP and PP amplification in humans. Improving the basal NO production may therefore be an attractive therapeutic goal in order to reduce the central BP load and to lower the cardiovascular risk.

\section{Acknowledgements}

This study was funded by a grant from the Deutsche Forschungsgemeinschaft to M.P.S. and R.E.S. (SFB 423, TP B5) and supported by a Research Fellowship from the European Society of Hypertension to M.P.S. We gratefully acknowledge the expert technical assistance of Ingrid Fleischmann, Dorothea BaderSchmieder, Simone Pejkovic, Susanne Avendano, Ulrike Heinritz and Ortrun Alter.

\section{References}

1 O’Rourke M, Kelly R, Avolio AP: The Arterial Pulse. Philadelphia, Lea \& Fibiger, 1992.

2 Weber T, Auer J, O’Rourke MF, Kvas E, Lassnig E, Lamm G, Stark N, Rammer M, Eber B: Increased arterial wave reflections predict severe cardiovascular events in patients undergoing percutaneous coronary interventions. Eur Heart J 2005;26:2657-2663.

3 Roman MJ, Devereux RB, Kizer JR, Lee ET, Galloway JM, Ali T, Umans JG, Howard BV: Central pressure more strongly relates to vascular disease and outcome than does brachial pressure: the Strong Heart Study. Hypertension 2007;50:197-203.
4 London GM, Asmar RG, O'Rourke MF, Safar ME: Mechanism(s) of selective systolic blood pressure reduction after a low-dose combination of perindopril/indapamide in hypertensive subjects: comparison with atenolol. J Am Coll Cardiol 2004;43:92-99.

5 Williams B, Lacy PS, Thom SM, Cruickshank K, Stanton A, Collier D, Hughes AD, Thurston H, O'Rourke M: Differential impact of blood pressure-lowering drugs on central aortic pressure and clinical outcomes: principal results of the Conduit Artery Function Evaluation (CAFE) study. Circulation 2006;113:1213-1225.
6 McEniery CM, Wallace S, Mackenzie IS, McDonnell B, Yasmin, Newby DE, Cockcroft JR, Wilkinson IB: Endothelial function is associated with pulse pressure, pulse wave velocity, and augmentation index in healthy humans. Hypertension 2006;48:602-608.

7 Ghiadoni L, Virdis A, Magagna A, Taddei S, Salvetti A: Effect of the angiotensin II type 1 receptor blocker candesartan on endothelial function in patients with essential hypertension. Hypertension 2000;35:501-506. 
8 Klingbeil AU, John S, Schneider MP, Jacobi J, Handrock R, Schmieder RE: Effect of AT1 receptor blockade on endothelial function in essential hypertension. Am J Hypertens 2003; 16:123-128.

9 Wilkinson IB, MacCallum H, Cockcroft JR, Webb DJ: Inhibition of basal nitric oxide synthesis increases aortic augmentation index and pulse wave velocity in vivo. Br J Clin Pharmacol 2002;53:189-192.

10 Pauca AL, O'Rourke MF, Kon ND: Prospective evaluation of a method for estimating ascending aortic pressure from the radial artery pressure waveform. Hypertension 2001; 38:932-937.

11 Levy BI, Benessiano J, Poitevin P, Safar ME: Endothelium-dependent mechanical properties of the carotid artery in WKY and SHR: role of angiotensin-converting enzyme inhibition. Circ Res 1990;66:321-328.

12 Boutouyrie P, Bezie Y, Lacolley P, Challande P, Chamiot-Clerc P, Benetos A, de la Faverie JF, Safar M, Laurent S: In vivo/in vitro comparison of rat abdominal aorta wall viscosity: influence of endothelial function. Arterioscler Thromb Vasc Biol 1997;17:13461355.

13 Fitch RM, Vergona R, Sullivan ME, Wang YX: Nitric oxide synthase inhibition increases aortic stiffness measured by pulse wave velocity in rats. Cardiovasc Res 2001;51:351358.

14 Kameyama H, Takeda K, Kusaba T, Narumiya $\mathrm{H}$, Tanda S, Kuwahara N, Yamada K, Tamagaki K, Okigaki M, Hatta T, Sasaki S: Augmentation of pulse wave velocity precedes vascular structural changes of the aorta in rats treated with $\mathrm{N}(\mathrm{o})$-nitro-L-arginine methyl ester. Hypertens Res 2005;28:439445.

15 Wilkinson IB, Qasem A, McEniery CM, Webb DJ, Avolio AP, Cockcroft JR: Nitric oxide regulates local arterial distensibility in vivo. Circulation 2002;105:213-217.

16 Ceravolo R, Maio R, Pujia A, Sciacqua A, Ventura G, Costa MC, Sesti G, Perticone F: Pulse pressure and endothelial dysfunction in never-treated hypertensive patients. J Am Coll Cardiol 2003;41:1753-1758.

17 Gullu H, Erdogan D, Caliskan M, Tok D, Yildirim E, Ulus T, Turan Sezgin A, Muderrisoglu $\mathrm{H}$ : Interrelationship between noninvasive predictors of atherosclerosis: transthoracic coronary flow reserve, flow-mediated dilation, carotid intima-media thickness, aortic stiffness, aortic distensibility, elastic modulus, and brachial artery diameter. Echocardiography 2006;23:835-842.

18 Jadhav UM, Kadam NN: Non-invasive assessment of arterial stiffness by pulse-wave velocity correlates with endothelial dysfunction. Indian Heart J 2005;57:226-232.

19 Kobayashi K, Akishita M, Yu W, Hashimoto $\mathrm{M}$, Ohni M, Toba K: Interrelationship between non-invasive measurements of atherosclerosis: flow-mediated dilation of bra- chial artery, carotid intima-media thickness and pulse wave velocity. Atherosclerosis 2004;173:13-18.

20 Nigam A, Mitchell GF, Lambert J, Tardif JC: Relation between conduit vessel stiffness (assessed by tonometry) and endothelial function (assessed by flow-mediated dilatation) in patients with and without coronary heart disease. Am J Cardiol 2003;92:395-399.

21 Pannier B, Guerin AP, Marchais SJ, Metivier F, Safar ME, London GM: Postischemic vasodilation, endothelial activation, and cardiovascular remodeling in end-stage renal disease. Kidney Int 2000;57:1091-1099.

22 Kinlay S, Creager MA, Fukumoto M, Hikita H, Fang JC, Selwyn AP, Ganz P: Endothelium-derived nitric oxide regulates arterial elasticity in human arteries in vivo. Hypertension 2001;38:1049-1053.

23 Ramsey MW, Goodfellow J, Jones CJ, Luddington LA, Lewis MJ, Henderson AH: Endothelial control of arterial distensibility is impaired in chronic heart failure. Circulation 1995;92:3212-3219.

24 Tesauro M, Thompson WC, Rogliani P, Qi L, Chaudhary PP, Moss J: Intracellular processing of endothelial nitric oxide synthase isoforms associated with differences in severity of cardiopulmonary diseases: cleavage of proteins with aspartate vs. glutamate at position 298. Proc Nat Acad Sci USA 2000;97: 2832-2835.

25 Fairchild TA, Fulton D, Fontana JT, Gratton JP, McCabe TJ, Sessa WC: Acidic hydrolysis as a mechanism for the cleavage of the Glu(298) $\rightarrow$ Asp variant of human endothelial nitric-oxide synthase. J Biol Chem 2001; 276:26674-26679.

26 Veldman BA, Spiering W, Doevendans PA, Vervoort G, Kroon AA, de Leeuw PW, Smits P: The GLU298ASP polymorphism of the NOS 3 gene as a determinant of the baseline production of nitric oxide. J Hypertens 2002; 20:2023-2027.

27 Guzik TJ, Black E, West NE, McDonald D, Ratnatunga C, Pillai R, Channon KM: Relationship between the G894T polymorphism (Glu298Asp variant) in endothelial nitric oxide synthase and nitric oxide-mediated endothelial function in human atherosclerosis. Am J Med Genet 2001;100:130-137.

28 Schneider MP, Erdmann J, Delles C, Fleck E, Regitz-Zagrosek V, Schmieder RE: Functional gene testing of the Glu298Asp polymorphism of the endothelial NO synthase. J Hypertens 2000;18:1767-1773.

29 Schneider MP, Delles C, Klingbeil AU, Ludwig M, Kolloch RE, Krekler M, Stumpe KO, Schmieder RE: Effect of angiotensin receptor blockade on central haemodynamics in essential hypertension: results of a randomised trial. J Renin Angiotensin Aldosterone Syst 2008;9:49-56

30 Klingbeil AU, John S, Schneider MP, Jacobi J, Weidinger G, Schmieder RE: AT1-receptor blockade improves augmentation index: a double-blind, randomized, controlled study. J Hypertens 2002;20:2423-2428

31 Schiffrin EL, Park JB, Pu Q: Effect of crossing over hypertensive patients from a $\beta$ blocker to an angiotensin receptor antagonist on resistance artery structure and on endothelial function. J Hypertens 2002;20: 71-78.

32 Schiffrin EL, Pu Q, Park JB: Effect of amlodipine compared to atenolol on small arteries of previously untreated essential hypertensive patients. Am J Hypertens 2002;15: 105-110.

33 Schiffrin EL, Park JB, Intengan HD, Touyz RM: Correction of arterial structure and endothelial dysfunction in human essential hypertension by the angiotensin receptor antagonist losartan. Circulation 2000;101: 1653-1659.

34 Taddei S, Virdis A, Ghiadoni L, Magagna A, Favilla S, Pompella A, Salvetti A: Restoration of nitric oxide availability after calcium antagonist treatment in essential hypertension. Hypertension 2001;37:943-948.

35 Von zur Mühlen B, Kahan T, Hagg A, Millgard J, Lind L: Treatment with irbesartan or atenolol improves endothelial function in essential hypertension. J Hypertens 2001;19: 1813-1818.

36 Tzemos N, Lim PO, MacDonald TM: Nebivolol reverses endothelial dysfunction in essential hypertension: a randomized, doubleblind, crossover study. Circulation 2001;104: 511-514.

37 Mahmud A, Feely J: Beta-blockers reduce aortic stiffness in hypertension but nebivolol, not atenolol, reduces wave reflection. Am J Hypertens 2008;21:663-667.

38 Michelson G, Warntges S, Harazny J, Oehmer S, Delles C, Schmieder RE: Effect of NOS inhibition on retinal arterial and capillary circulation in early arterial hypertension. Retina 2006;26:437-444.

39 Schneider MP, Klingbeil AU, Delles C, Schmidt BM, John S, Schmieder RE: Vitamin $\mathrm{C}$ augments the renal response to $\mathrm{L}$-arginine in smokers. Nephrol Dial Transplant 2003; 18:1512-1517.

40 Gamboa A, Shibao C, Diedrich A, Choi L, Pohar B, Jordan J, Paranjape S, Farley G, Biaggioni I: Contribution of endothelial nitric oxide to blood pressure in humans. Hypertension 2007;49:170-177.

41 Stewart AD, Millasseau SC, Kearney MT, Ritter JM, Chowienczyk PJ: Effects of inhibition of basal nitric oxide synthesis on carotid-femoral pulse wave velocity and augmentation index in humans. Hypertension 2003; 42:915-918

42 Wilkinson IB, MacCallum H, Hupperetz PC, van Thoor CJ, Cockcroft JR, Webb DJ: Changes in the derived central pressure waveform and pulse pressure in response to angiotensin II and noradrenaline in man. J Physiol 2001;530:541-550. 